\title{
Inhibition of SHP2 in basal-like and triple-negative breast cells induces basal-to-luminal transition, hormone dependency, and sensitivity to anti-hormone treatment
}

\author{
Hua Zhao and Yehenew M Agazie*
}

\begin{abstract}
Background: The Src homology phosphotyrosyl phosphatase 2 (SHP2) is a positive effector of cell growth and survival signaling as well transformation induced by multiple tyrosine kinase oncogenes. Since the basal-like and triple-negative breast cancer (BTBC) is characterized by dysregulation of multiple tyrosine kinase oncogenes, we wanted to determine the importance of SHP2 in BTBC cell lines.

Methods: Short hairpin RNA-based and dominant-negative expression-based SHP2 inhibition techniques were used to interrogate the functional importance of SHP2 in BTBC cell biology. In addition, cell viability and proliferation assays were used to determine hormone dependency for growth and sensitivity to anti-estrogen treatment.

Results: We show that inhibition of SHP2 in BTBC cells induces luminal-like epithelial morphology while suppressing the mesenchymal and invasive property. We have termed this process as basal-to-luminal transition (BLT). The occurrence of BLT was confirmed by the loss of the basal marker alpha smooth muscle actin and the acquisition of the luminal marker cytokeratin 18 (CK18) expression. Furthermore, the occurrence of BLT led to estrogen receptor alpha (ERa) expression, hormone dependency, and sensitivity to tamoxifen treatment.

Conclusions: Our data show that inhibition of SHP2 induces BLT, ERa expression, dependency on estrogen for growth, and sensitivity to anti-hormone therapy. Therefore, inhibition of SHP2 may provide a therapeutic benefit in basal-like and triple-negative breast cancer.
\end{abstract}

Keywords: SHP2, ERa, Breast cancer, Invasiveness, Basal-to-luminal transition, Tamoxifen

\section{Background}

The recent decline in breast cancer death rate is attributed, at least in part, to availability of targeted therapies such as Herceptin against HER2-positive and tamoxifen against estrogen receptor-positive breast cancers [1]. Unfortunately, no such treatment options exist for the basal-like and/or triple-negative breast cancer (BTBC). As a result, BTBC causes disproportionately high mortalities in women [2], mainly in African-American women and in younger women of all ethnicities. The term basal-like was derived from the expression profile of basal cytokeratins (CK5/6, CK14 and CK17) by BTBC tumors, proteins

\footnotetext{
* Correspondence: yagazie@hsc.wvu.edu

Department of Biochemistry and The Marry Babb Randolph Cancer Center School of Medicine, West Virginia University, Morgantown, WV 26506, USA
}

expressed by the basal cells of the normal breast, the myoepithelial cells $[1,3]$. But, recent reports suggest that BTBC may also originate from pluripotent luminal cells [4]. Another characteristic feature of BTBC tumors is the elevated expression of the epidermal growth factor receptor (EGFR) and multiple other receptor tyrosine kinases (RTKs), including the MET, the FGFR, and the IGF-1R [5-8].

The Src homology phosphotyrosyl phosphatase 2 (SHP2) is an essential transducer of mitogenic and cell survival signaling downstream of multiple RTKs, including those dysregulated in BTBC [9-11]. In addition, SHP2 is important for cell transformation induced by oncogenic RTKs and v-Src [12-15]. It was thus reasonable to determine the importance of SHP2 in BTBC cell lines in which multiple RTKs are known to be dysregulated. SHP2 is composed of 
two Src homology 2 domains in the N-terminal and a PTP domain in the C-terminal regions $[16,17]$. The SH2 domains allow interaction with phosphotyrosine while the PTP domain dephosphorylates target substrates. In a resting state or in the absence of tyrosine kinase signaling, SHP2 assumes a closed inactive confirmation due to intramolecular interaction between the $\mathrm{N}$-terminal $\mathrm{SH} 2$ and the PTP domains. The binding of the SH2 domains to phosphotyrosine disrupts the intramolecular interaction, leading to an open and active confirmation. Hence, increased tyrosine kinase signaling induced by dysregulated RTKs in BTBC can lead to increased SHP2 activity and augmented downstream signaling. In this report, we show that inhibition of SHP2 in BTBC cells reverses the mesenchymal phenotype, abolishes invasiveness, induces basal-to-luminal transition (BLT), and confers hormone dependency and sensitivity to anti-hormone (tamoxifen) treatment.

\section{Methods}

\section{Cells, cell culture and reagents}

The MDA-MB231 and the MDA-MB468 breast cancer cell lines and the MCF-10A cells were purchased from ATCC. These cells were grown as described previously $[18,19]$. The anti- $\beta$-actin monoclonal antibody (A5441) was from SigmaAldrich, the anti-Snail antibody (SN9H2) was from Cell Signaling, the anti-EGFR antibody (610017) was from BD Biosciences, the anti CK18 antibody (M7010) was from DAKO, the anti-smooth muscle actin (MA1-26017) and the anti-estrogen receptor alpha (MA1-310) antibodies were from Thermo Scientific, and the anti-MMP2 (MAB3308) and the anti-MMP9 (AB13458) antibodies were from Millipore. The anti-SHP2 (SC-7384), the anti-vimentin (SC32322), the anti-progesterone receptor (SC-538), and the anti-fibronectin (SC-18825) antibodies were from Santa Cruz Biotechnology. Anti-mouse and anti-rabbit secondary antibodies conjugated with horseradish peroxidase were purchased from Jackson Immuno-Research Laboratories.

\section{Inhibition of SHP2 by shRNA and by dominant-negative expression}

Two independent shRNA sequences (double-stranded deoxyoligonucleotides) previously shown to be specific for SHP2 $[18,20,21]$ were used for silencing of SHP2 in the MDA-MB231 and MDA-MB468 cells. A short hairpin RNA against luciferase was used as a control as also described previously [18].

\section{Preparation of cell lysates and immunostaining analyses}

Cell lysates were prepared in a buffer containing $20 \mathrm{mM}$ Tris-HCl, pH7.2, $150 \mathrm{mM} \mathrm{NaCl}, 50 \mathrm{mM} \mathrm{NaF}, 1 \mathrm{mM}$ EDTA, $10 \%$ glycerol, $1 \%$ triton-X-100, $1 \mathrm{mM}$ sodium orthovanadate and a protease inhibitor cocktail. For total cell lysate analysis, proteins were separated using a standard polyacrylamide gel electrophoresis, transferred onto nitrocellulose membranes, blocked in 3\% bovine serum albumin, stained with primary antibodies overnight at $4^{\circ} \mathrm{C}$, washed three times with TBST (Tris-buffered saline containing 0.1\% Tween-20) and incubated with secondary antibodies for 1 hour at room temperature. Finally, stained bands were detected by the chemiluminescence method.

\section{D monolayer wounding and 3D laminin-rich basement membrane (LRBM) culture}

For monolayer wounding assay, cells were grown in DMEM containing 10\% FBS and scratched with a pipet tip to create a gap. The ability of the cells to migrate and fill the space was monitored under a microscope. Pictures were collected under an IX-71 Olympus microscope using the $5 \times$ objective immediately after wounding and every 12 hours thereafter.

For 3D invasion assay, a modified version of the lamininrich basement membrane (LRBM) culture, sometimes referred to as 3D matrigel assay, was used. The basic protocol has been previously reported [22]. Four-well chamber slides (Falcon) were overlaid with $80 \mu \mathrm{l}$ of growth factor reduced LRBM medium (BD Biosciences) containing $10 \mu \mathrm{g} / \mathrm{ml} \mathrm{DQ}$ collagen and allowed to solidify for 1 hour at $37^{\circ} \mathrm{C}$. Approximately $10^{3}$ cells suspended in regular growth medium were seeded per well and protease activity was determined under a fluorescent microscope after 24 hours of incubation. Light and fluorescent pictures were collected under Olympus IX71 microscope with attached CCD camera. Fluorescent intensity measurement of collagen degradation was performed using the Olympus Microsuite software.

For protease expression and secretion studies, the same LRBM culture system, lacking the DQ collagen, was used. In addition, cells were serum starved overnight (fed with serum-free DMEM) before harvesting the conditioned medium. Corresponding total cell lysates were used for determining intracellular protein levels.

To determine the effect of SHP2 inhibition on differentiation of BTBC cells, the LRBM culture described previously $[19,23]$ was used. Approximately $10^{3}$ cells were suspended in $250 \mu \mathrm{l}$ of assay medium (DMEM supplemented with $2 \%$ horse serum, $10 \mu \mathrm{g} / \mathrm{ml}$ recombinant human insulin, $0.5 \mu \mathrm{g} / \mathrm{ml}$ hydrocortisone, $5 \mathrm{ng} / \mathrm{ml} \mathrm{EGF}$, $100 \mathrm{ng} / \mathrm{ml}$ cholera toxin and $1 \mathrm{X}$ penicillin/streptomycin) and seeded on a solidified LRBM and cultured at $37^{\circ} \mathrm{C}$ in $7 \% \mathrm{CO}_{2}$ incubator. The cells were re-fed with assay medium every 4 days and phase contrast pictures were taken after 15 days.

\section{Hormone dependency and tamoxifen sensitivity}

Cells were seeded in 96-well plates in DMEM containing $10 \%$ FBS plus or minus estradiol (E2) for 48 hours. The effect of E2 on cell growth was determined using a Luminescent cell viability assay (Promega) that measures growth based on ATP levels. The manufacturer's protocol 
was followed in seeding cells, preparation of reagents, and luminescent measurements. For testing sensitivity to tamoxifen, cells were grown in the same way for 24 hours and then treated with vehicle (DMSO) or $5 \mu \mathrm{M}$ tamoxifen for another 24 hours. Cell growth was measured in Synergen H3 (Biotech) plate reader and the data was analyzed with the IGEN-5 software.

\section{Results}

SHP2 is essential for the maintenance of the mesenchymal morphology in BTBC cells

To determine the functional significance of SHP2 in BTBC, we chose the MDA-MB231 and the MDAMB468 breast cancer cell lines which are known to be basal-like and triple-negative [24,25]. These cells harbor several genetic abnormalities commonly discovered in $\mathrm{BTBC}$, including activating Ras mutation in the MDAMB231, elevated EGFR expression and p53 mutation in both $[24,26]$, and PTEN homo-deletion and EGFR gene amplification in the MDA-MB468 cells [24,27]. In addition, both cell lines show elevated SHP2 expression [28]. Hence, they are appropriate for testing the importance of SHP2 in BTBC. The expression of SHP2 was silenced with two independent shRNA sequences that were previously shown to be specific and devoid of offtarget effects $[18,21]$. Cells expressing luciferase shRNA were used as controls in this study. As shown in Figure 1A, each shRNA effectively silenced SHP2 expression. Microscopic examination of confluent cells grown in 2D cultures revealed that the SHP2 silenced cells had acquired an epithelial morphology characteristic of luminal epithelial cells while the parental and the control cells showed the expected elongated and spindle-shaped mesenchymal morphology. In other words, silencing SHP2 expression induced morphological changes that are comparable to the MCF-10 cells, the immortalized and non-tumorigenic breast epithelial cells commonly used as "normal" controls (Figure 1B). Morphological changes induced by SHP2 silencing were obvious even in non-confluent cells (Additional file 1: Figure S1A). Similar results were obtained in the MDAMB468 cells (Additional file 1: Figure S1B and C).

To further confirm that the PTPase activity of SHP2 was responsible for the observed morphological changes and to rule out the possibility of any off-target shRNA effects, we inhibited SHP2 function by expression of the PTPase-dead SHP2 (C459S-SHP2), also known as dominant-negative SHP2, using the strategy described by us previously $[12,13]$. As shown in Figure 1C, FLAGtagged wild-type SHP2 (WT-SHP2) and C459S-SHP2 were efficiently expressed in both cells lines. In agreement with shRNA-based SHP2 inhibition, expression of C459S-SHP2 led to similar morphological changes, while the wild-type counterpart (WT-SHP2) or vector alone did not (Figure 1D). These results suggest that SHP2 is required for the maintenance of the mesenchymal property in BTBC cells and its inhibition induces luminallike morphology in BTBC cells.

\section{SHP2 is essential for the migratory and invasive properties of BTBC cells}

Most BTBC cell lines, including the cells used in this study are highly mesenchymal and exhibit enhanced migratory and invasive properties [29]. The morphological changes induced by SHP2 inhibition were indicative of the importance of SHP2 in these properties. To test this possibility, we employed the simple wound-healing assay in which cells will be induced to migrate and fill the space created by scratching. In agreement with our previous report on effect of SHP2 in cell polarity [18], silencing SHP2 expression retarded cell migration. While the control cells were able to heal the wound in 24 hours, the SHP2-silenced cells were unable to do so even in 48 hours (Figure 2A and B). Similar results were obtained when SHP2 function was inhibited by expression of C459S-SHP2 (Additional file 2: Figure S2A and B). Hence, SHP2 is essential for the migratory behavior of BTBC cells.

The effect of SHP2 inhibition on the invasive property of BTBC cells was tested by the DQ matrigel assay. This assay system utilizes collagen IV conjugated to quenchedFITC, which fluoresces upon collagen degradation. Fluorescence emission is proportional to amount of collagen degradation, and therefore, it can be used to measure ability of cells to degrade and invade extracellular matrix $(\mathrm{ECM})$. Cells were mixed with laminin-rich basement membrane (LRBM), containing approximately $10 \mu \mathrm{g} / \mathrm{ml}$ DQ collagen as described previously [22], and seeded in slide culture chambers. ECM degradation was monitored under a fluorescent microscope as described in the materials and methods. While the parental and the control cells showed enhanced matrigel degradation, the SHP2silenced cells had very little or no degradation (Figure 2C and $\mathrm{E})$. The corresponding light pictures further confirmed the highly mesenchymal and invading nature of the parental and control cells and the absence of such phenotypes in the SHP2-silened cells. Fluorescence intensity measurements further confirmed that the parental and the control cells degraded collagen matrices approximately 20 times more than the SHP2-silenced cells (Figure 2D and F). Expression of C459S-SHP2 also led to suppression of the invasive phenotype (Additional file 2: Figure S2C). These results clearly demonstrated that the invasive property of BTBC cells is SHP2 dependent.

\section{SHP2 is required for expression of EMT proteins and matrix-degrading enzymes}

The retardation of cell migration and the blockade of invasiveness induced by SHP2 silencing implied loss of the 


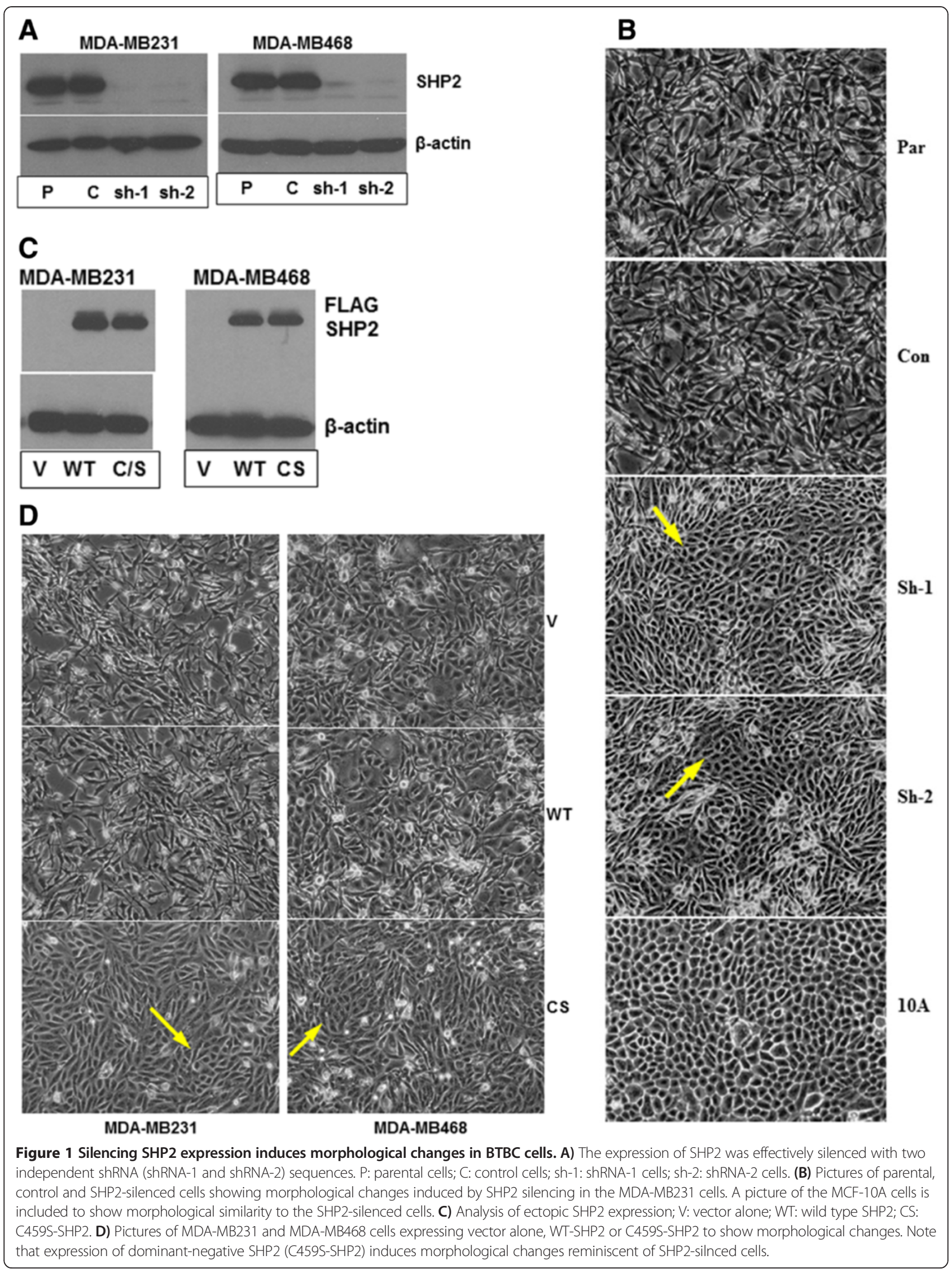



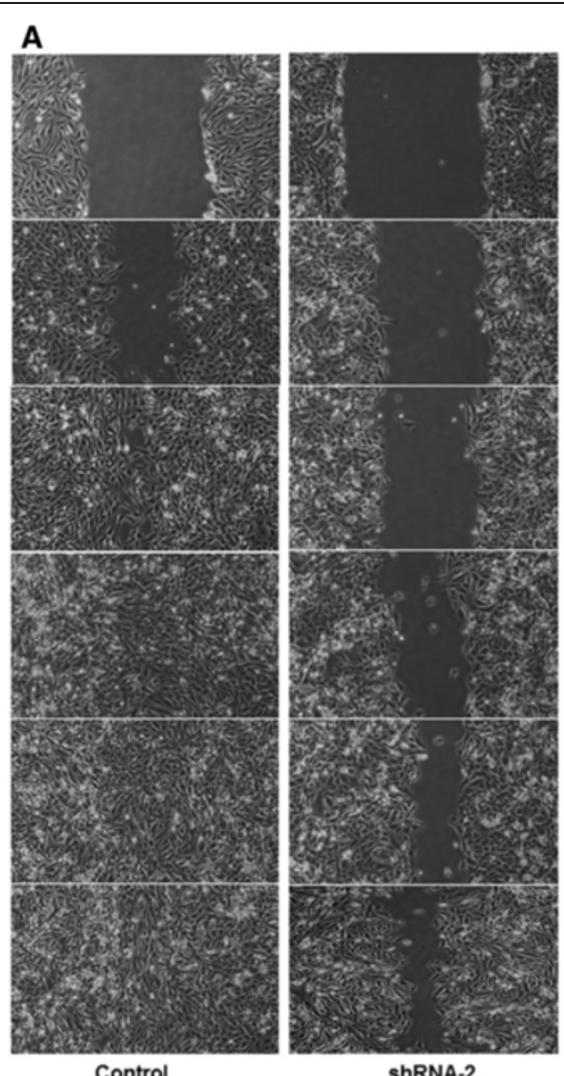

Start
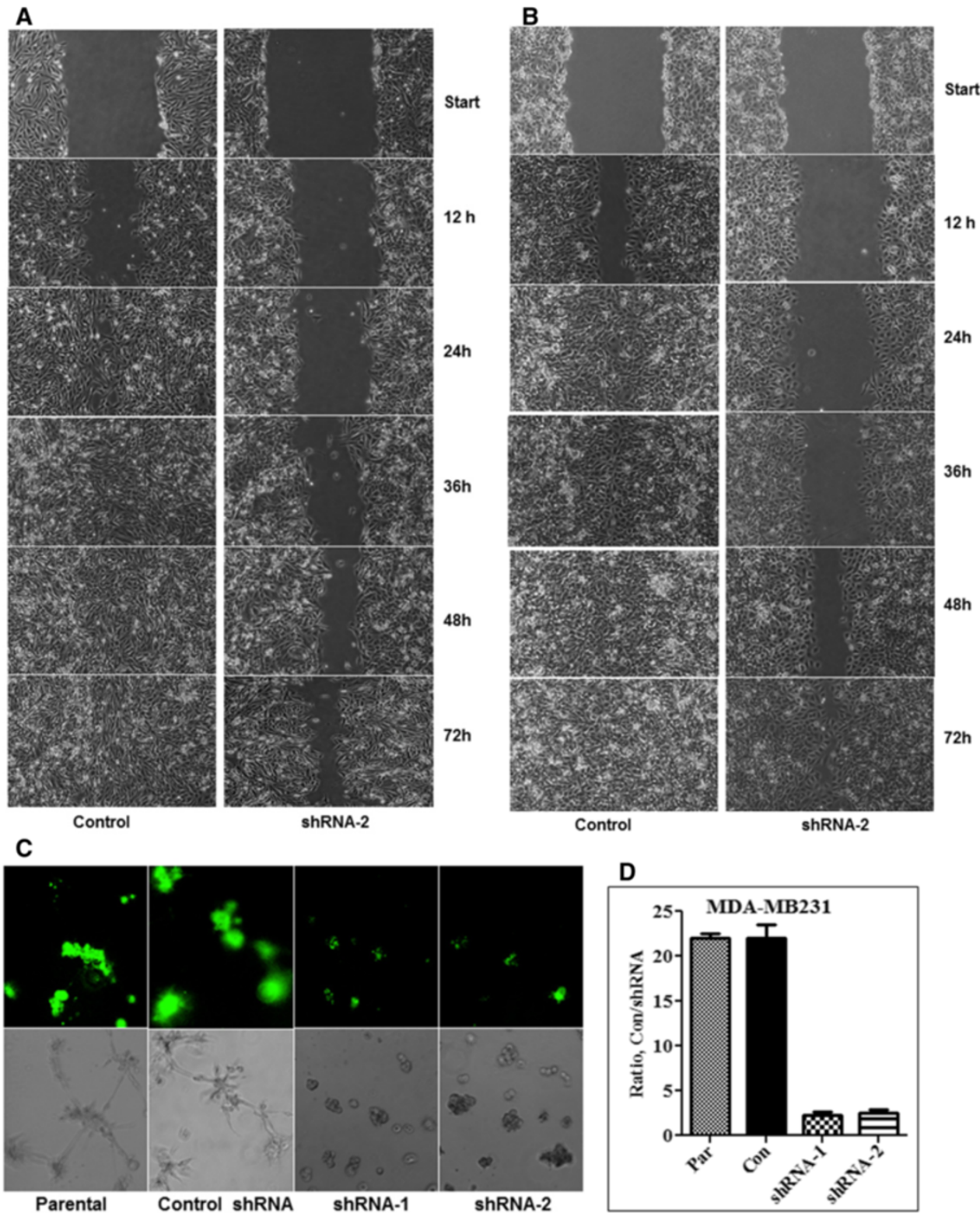

E
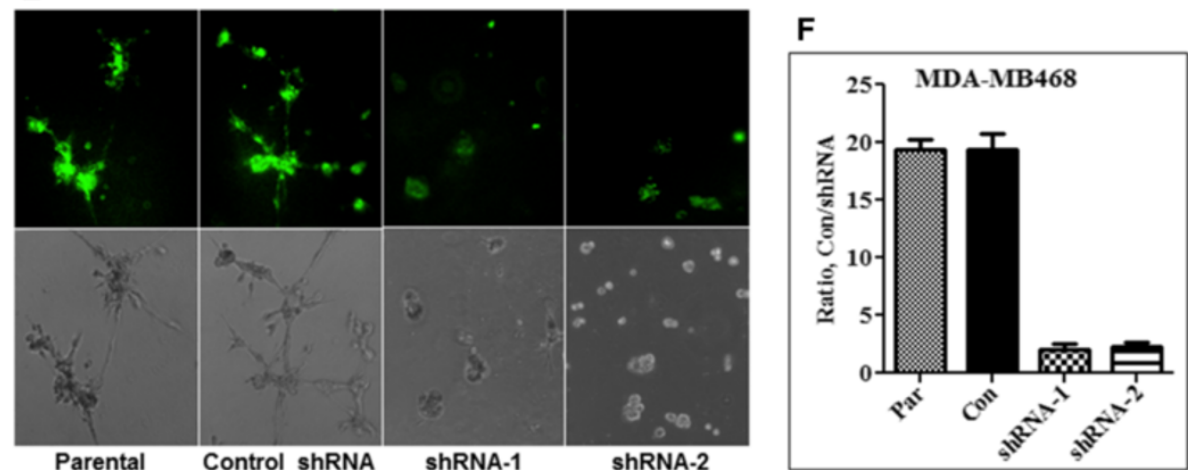

Figure 2 (See legend on next page.) 
(See figure on previous page.)

Figure 2 Effect of SHP2 silencing on cell migration. Silencing SHP2 expression retards cell migration in the MDA-MB231 (A) and the MDA-MB468 (B) cells. Data shown was from the control and the shRNA-2 cells derived from the respective cell lines. C) Effect of SHP2 silencing on the ability of the MDA-MB231 cells to degrade FITC-labeled collagen in 3D matrigel invasion assay. D) Fluorescent intensity measurement of collagen degradation by the MDA-MB231 cells. E) Effect of SHP2 silencing on the ability of the MDA-MB468 cells to degrade FITC-labeled collagen in 3D matrigel invasion assay. F) Fluorescent intensity measurement of collagen degradation by the MDA-MB468 cells. Data shown was mean \pm SEM of three independent experiments.

mesenchymal phenotype. To further validate these observations, total cell lysates prepared from the 3D matrigel cultures were analyzed for expression of the mesenchymal markers, Snail, fibronectin (FN), and Vimentin. Consistent with the observed phenotypic changes, the levels of Snail, FN, and Vimentin were significantly reduced in the SHP2-silenced cells (Figure 3A), confirming that SHP2 is essential for the maintenance of the mesenchymal property in BTBC cells. Assuming that one of the mechanisms for SHP2 in promoting the expression of EMT proteins could be through protein stability, we conducted proteasome inhibition studies with Mg-132 treatment. As shown in Figure 3B, treating cells with Mg-132 restored FN and Snail, but not vimentin. These findings suggest that SHP2 promotes the stability of FN and Snail, but its positive effect on vimentin might be at the level of transcription.

The proteasome inhibition studies were suggestive of enhanced degradation of FN and Snail in the absence of SHP2. To examine the dynamics of FN and Snail degradation in the presence and absence of SHP2, we first restored these proteins by Mg-132 treatment for 6 hours. This was necessary since FN and Snail levels in the shRNA cells were low and hence could not be compared. After removal of Mg-132 by washing, new protein synthesis was blocked by cyclohexamide treatment for variable time points. As shown Figure $3 \mathrm{C}$ and $\mathrm{E}$, the levels of both proteins decreased very rapidly in the SHP2-silenced cells, but very slowly in the controls. Band density measurements revealed that the half-life of FN was less than 2 hours while that of Snail was less than 1 hour in the SHP2 silenced cells. But, the half-life of both proteins in the control cells was at least 6 hours (Figure 3D and F). Hence, loss of SHP2 destabilizes some EMT proteins in BTBC cells.

Previous studies have shown that the MDA-MB231 and the MDA-MB468 cells express MMP2 and MMP9 $[30,31]$. We reasoned that the loss of the invasive property in the SHP2-silenced cells could be related to loss of the expression of these proteases. We thus tested the impact of SHP2 inhibition on the expression and secretion of MMP2 and MMP9 in matrigel assays. Cells were seeded in matrigel and the conditioned media harvested from these cultures was analyzed by immunoblotting for MMP-2 and MMP-9. Inhibition of SHP2 blocked the secretion of MMP9 into the medium in both the MDAMB231 and the MDA-MB468 cells, although the level of
MMP9 in the control MDA-MB468 cells was relatively low in the first place (Figure 3G and H, top panels). Analysis of corresponding total cell lysates showed that the expression of MMP9 was reduced, but not completely blocked by SHP2 inhibition in both cells (Figure 3G and H, second panels). However, the level of MMP9 in the control MDA-MB468 cells was relatively low when compared to the control MDA-MB231 cells, which also reflects the low level of MMP9 secretion into the medium by the MDA-MB468 cells. The secretion and expression of MMP2 was also suppressed in the SHP2-silenced MDAMB231 cells (Figure 3G, third panel), but this protease was undetectable in the MDA-MB468 cells (data not shown). Reprobing for $\beta$-actin in the total cell lysates showed comparable level of protein loading. Overall, these results suggest that SHP2 promotes the invasive phenotype of BTBC cells by positively regulating the expression and secretion of MMP9 and possibly MMP2 in a cell context-dependent manner.

\section{Inhibition of SHP2 induces basal-to-luminal transition}

As shown in Figure 1 and Additional file 1: Figure S1, silencing SHP2 expression induced a luminal-like epithelial morphology in BTBC cells. Based on these findings, we reasoned that inhibition of SHP2 may induce conversion of BTBC cells to a luminal lineage, which we termed basalto-luminal transition (BLT). This possibility was tested by the laminin-rich basement membrane (LRBM) culture where non-transformed luminal breast epithelial cells like the MCF10A form acini-like structures while breast cancer cells produce a continuously-growing amorphous cellular mass $[19,23]$. As expected, the control cells formed a disorganized cellular mass that continued to grow and invade, whereas the SHP2-silenced cells grew very slowly, forming acini-like spheroid structures that are comparable to those formed by the control MCF-10A cells (Figure 4A and B). These results suggest that inhibition of SHP2 reverses the transformation and invasive phenotype and induces BLT in BTBC cells.

To further confirm the occurrence of BLT, the expressions of basal and luminal markers were analyzed by immunoblotting of total cell lysates prepared from LRBM cultures. The results showed that the expression of the basal marker $\alpha$-SMA (alpha smooth muscle actin) was higher in the parental and the control cells and lower in the corresponding SHP2-silenced cells (Figure 4C). But, the converse was true for expression of the luminal 


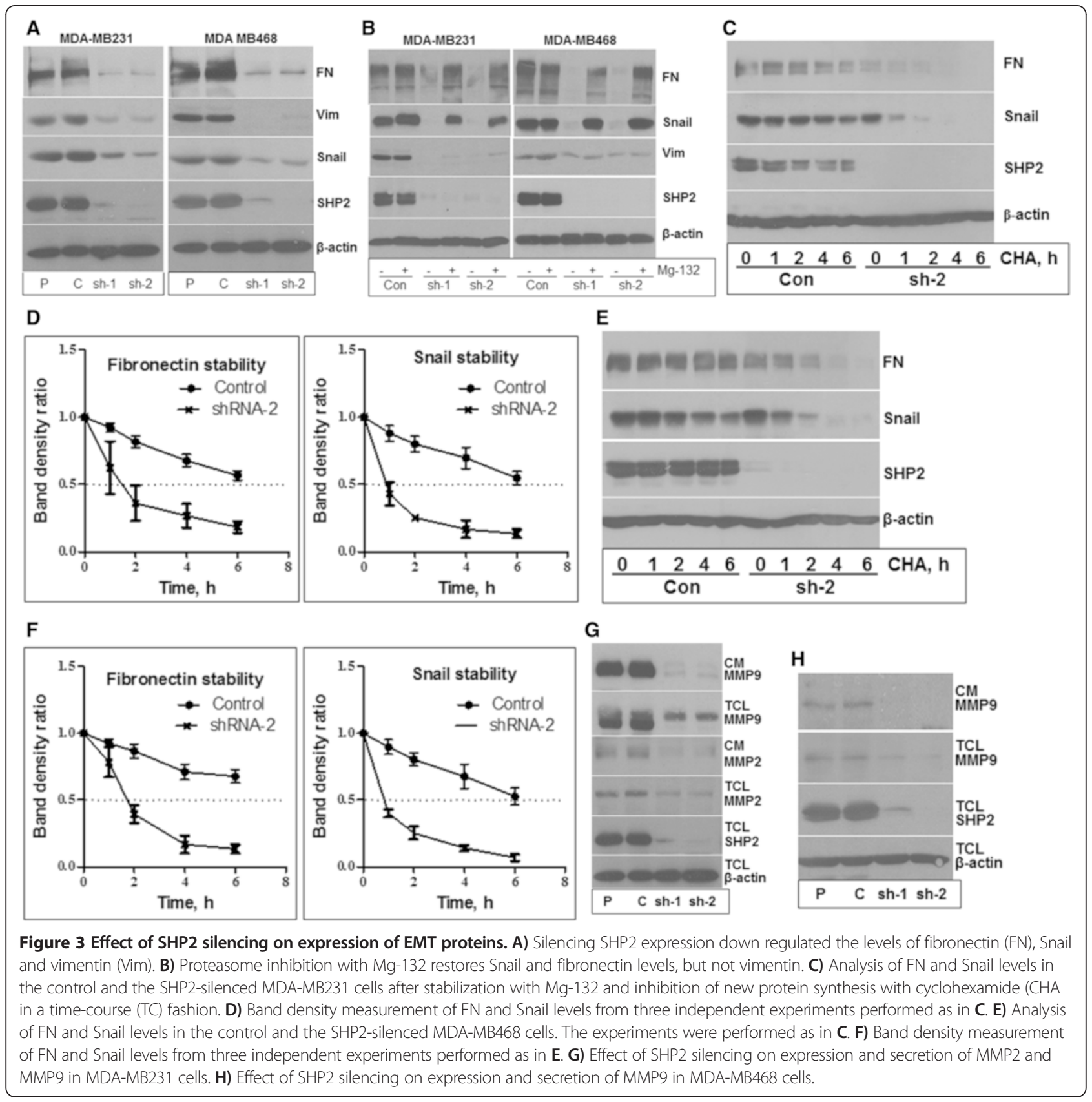

marker CK18 (cytokeratin 18). These results confirm that inhibition of SHP2 leads to BLT in triple-negative breast cancer cells.

The next logical step was to determine whether induction of BLT is accompanied by loss of the triple-negative status. Normal luminal breast epithelial cells and breast cancer cells that belong to the luminal subtype express the hormone receptors estrogen receptor alpha $(E R \alpha)$ and progesterone receptor (PR). We thus determined the state of ER $\alpha$ and PR expression in the same total cell lysates. Consistent with the occurrence of BLT, inhibition of SHP2 led to expression of ER $\alpha$ in triple-negative breast cancer cells (Figure 4C). However, we were unable to detect PR expression by immunoblotting with a specific antibody (not shown). Nonetheless, these findings demonstrate that SHP2 is essential for the maintenance of the triple-negative status in BTBC cells. In other words, inhibition of SHP2 in BTBC cells induces differentiation to hormone-positive cells.

\section{Inhibition of SHP2 induces estrogen dependency and sensitivity to tamoxifen}

The expression of ER $\alpha$ (Figure 4C) in SHP2-silenced cells suggested that they may depend on estrogen 

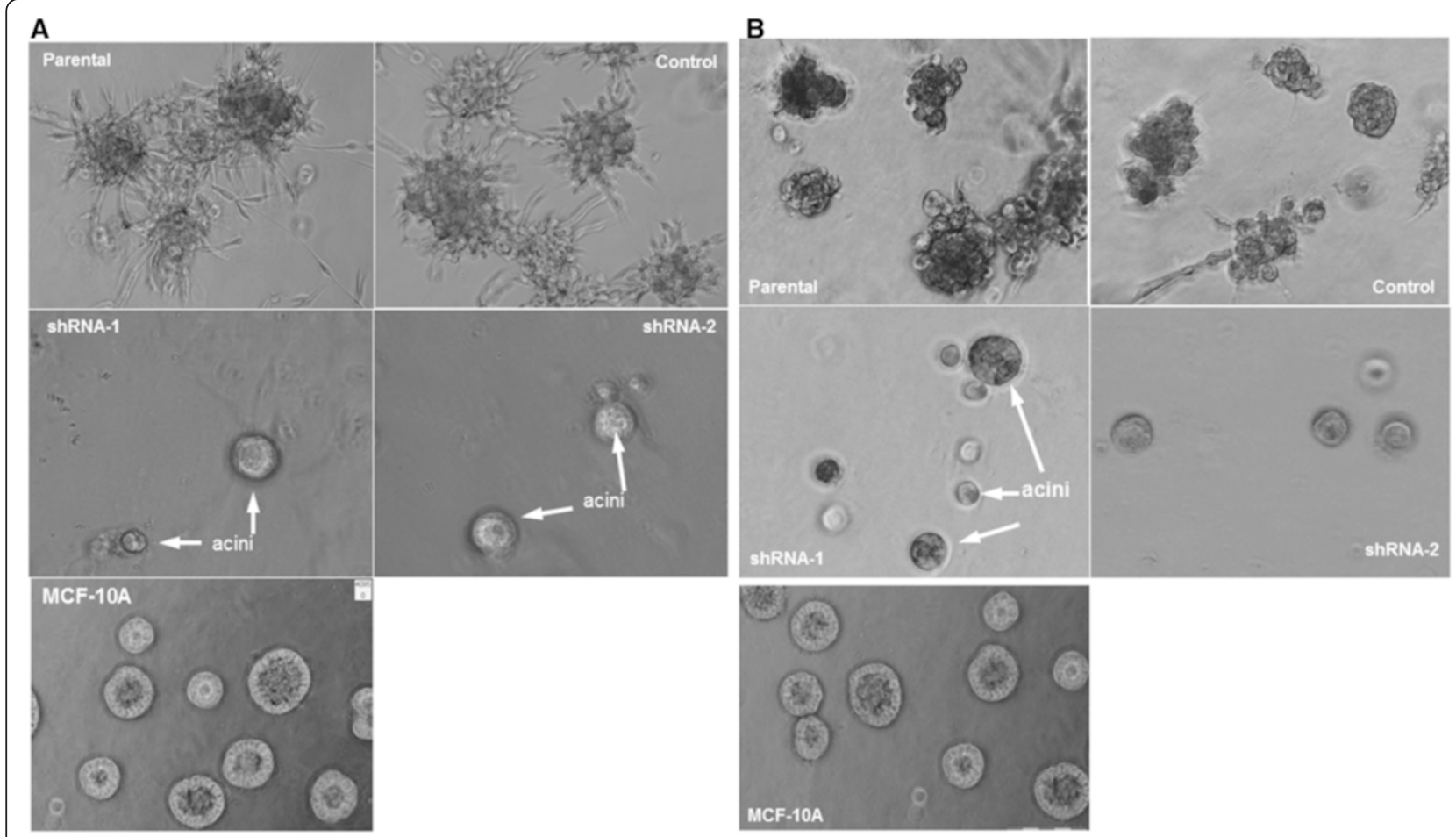

C

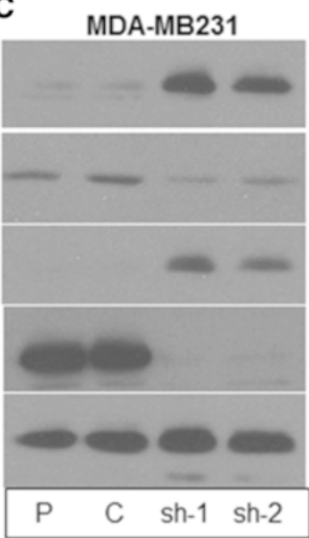

MDA-MB468

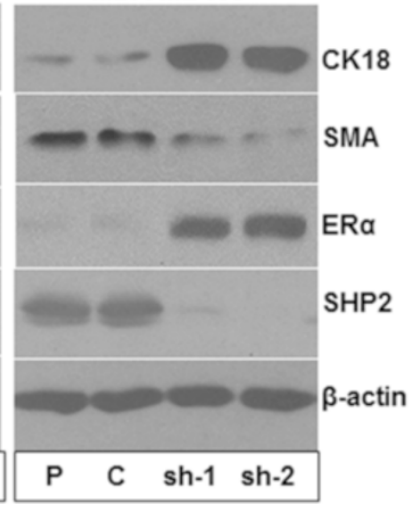

Figure 4 Silencing SHP2 expression induces acini-like structure formation. A) Light pictures of parental, control and SHP2-silenced MDA-MB231 cells cultured in 3D LRBM matrigel that allows acini-like structure formation. B) Light pictures of parental, control and SHP2-silenced MDA-MB468 cells cultured in 3D LRBM matrigel that allows acini-like structure formation. Pictures of acini-like structures formed by the MCF-10A cells cultured under identical conditions is shown in both $\mathbf{A}$ and $\mathbf{B}$ for comparison. C) Effect of SHP2 silencing on expression of basal and luminal markers and estrogen receptor alpha (ERa). Data presented is representative of at least three independent experiments.

signaling for growth. To test this possibility, we conducted cell growth studies in the presence and absence of estradiol (E2) using a cell viability assay (Promega) that determines cell growth based on ATP levels. The MCF-10A (non-transformed breast epithelial cells) and the MCF-7 (the hormone-positive breast cancer cell line) cells that require E2 for growth were used as positive controls. While the control cells did not respond to E2 treatment, the SHP2-silenced cells showed an approximately $30 \%$ increase in cell growth rate over the unstimulated counterparts (Figure 5A). As expected, the growth of the MCF-10A and the MCF-7 cells was retarded by E2 subtraction in the growth medium. We also determined the effect of E2 on cell growth rate by direct counting in a time-course fashion as described previously [32]. Consistent with the cell viability data, addition of E2 in the growth medium increased the growth rate of the SHP2-slinced cells. For instance, the unstimulated and the E2-stimulated SHP2-silenced cells grew by approximately 4-fold and 7-fold, respectively, over a period of 3 days (Figure 5B). As expected, the control cells grew by more than 10 -fold over the same 


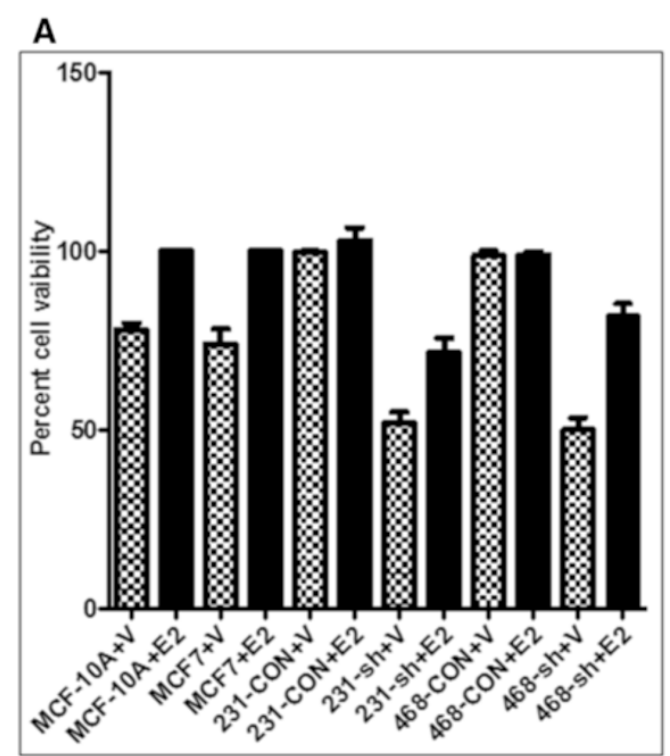

B
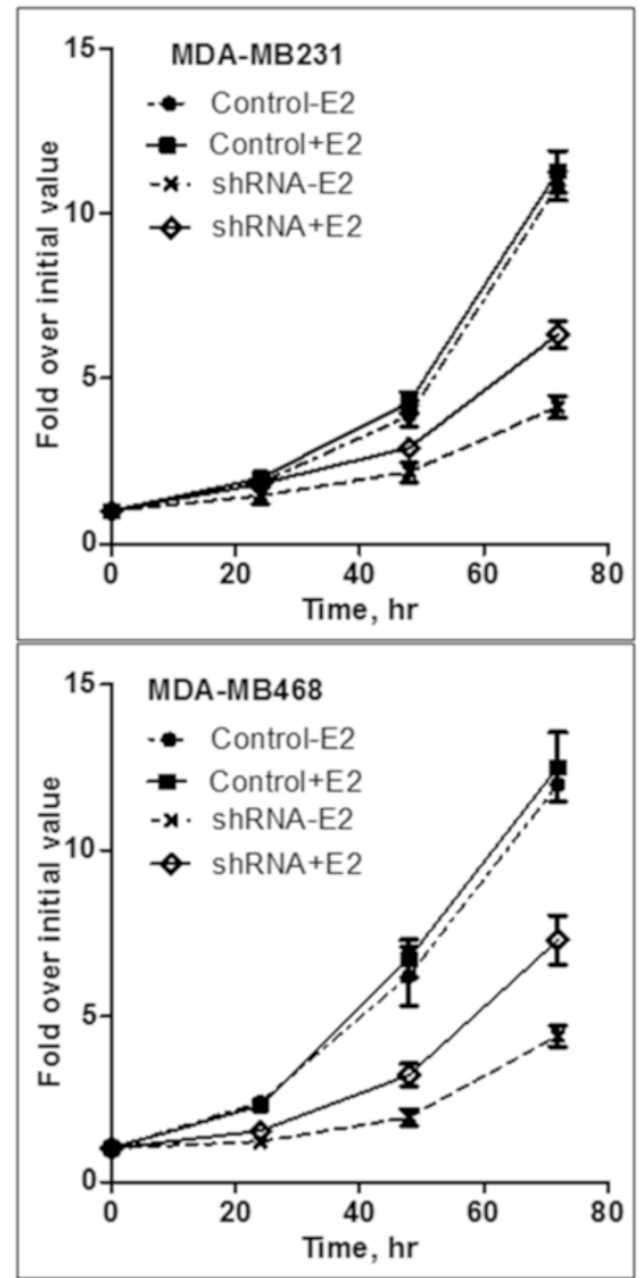

C

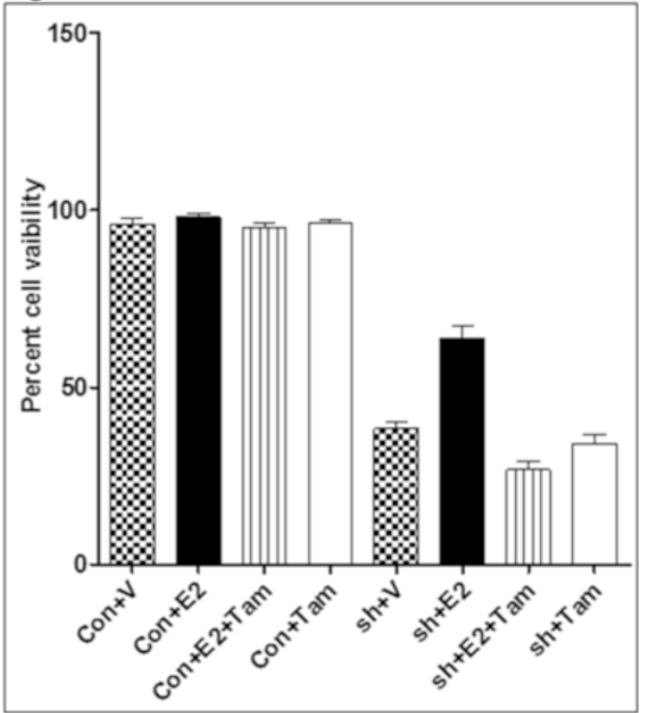

D

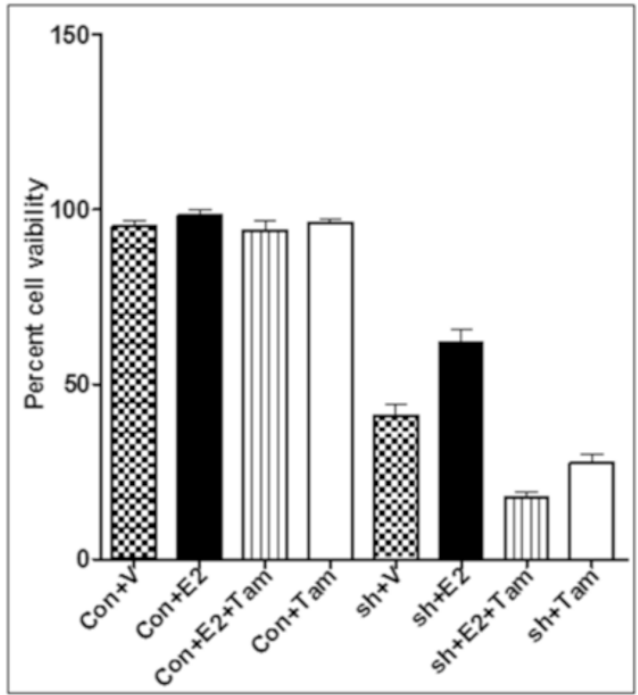

Figure $\mathbf{5}$ (See legend on next page.) 
(See figure on previous page.)

Figure 5 Effect of SHP2 silencing on estrogen responsiveness. A) Comparison of cells for responsiveness to estradiol treatment using cell viability assay. B) Effect of estradiol on the growth of the control and the SHP2-silenced MDA-MB231 and MDA-MB468 cells as determined by direct cell counting in a time-course fashion. C) Effect of SHP2 silencing on sensitivity to tamoxifen in the MDA-MB231 cells. D) Effect on SHP2 silencing on sensitivity to tamoxifen in the MDA-MB468 cells. V: vehicle; E2: estradiol; Tam: tamoxifen; Con: control shRNA; sh: SHP2 shRNA.

period of time. These observations suggest that SHP2 inhibition-induced ER $\alpha$ expression confers hormone dependency.

Based on the above findings, we reasoned that hormone dependency may lead to sensitivity to anti-hormone treatment. We used the same assay system to determine sensitivity to tamoxifen (Tam) by adding $1 \mu \mathrm{g} / \mathrm{ml}$ and incubation for 48 hours. While the control cells showed very little or no sensitivity, the SHP2 silenced cells exhibited loss of viability even below those that were not stimulated with E2 (Figure 5C and D). In other words, Tam abrogated the effect of E2 on cell growth in the SHP2silenced cells. However, treating SHP2-silenced cells with Tam alone did not induce further inhibition than that induced by SHP2 silencing. These findings further support our hypothesis that inhibition of SHP2 induces ER $\alpha$ expression, hormone dependency, and sensitivity to antihormone therapy.

\section{Discussion}

When compared to the hormone- and HER2-positive subtypes of breast cancer, BTBC causes disproportionately high mortalities in women $[2,33,34]$. The major factors are the aggressive nature of the disease and the lack of targeted therapies. Several RTKs, including EGFR, FGFR, c-MET, IGF-1R, and Eph2 are known to be dysregulated in $\mathrm{BTBC}[5,6,8]$, but development of targeted therapies against them has not been successful. SHP2 is a critical downstream signal transducer of many RTKs, including those dysregulated in BTBC $[9-11,13,35,36]$, but its functional role has not been effectively demonstrated. As a result, its potential for targeted therapy in BTBC has not been explored. In this study, we conducted shRNA-based inhibition studies to show the importance of SHP2 in BTBC.

Most BTBC cell lines, including the cells used in this study are highly mesenchymal and exhibit enhanced migratory and invasive properties [29]. We found that silencing SHP2 expression induces morphological changes that resemble luminal breast epithelial cells which are apparent even in non-confluent cells (Figure $1 \mathrm{~B}$ and Additional file 1: Figure S1). Expression of the PTPasedead (dominant-negative) SHP2 also induced similar phenotypes (Figure 1D), confirming that SHP2 is essential for the mesenchymal morphology of BTBC cells. These findings are consistent with our previous report in which we showed the importance of SHP2 for the maintenance of the transformed phenotype in HER2-positive and basal-like breast cancer cells [19]. Therefore, SHP2 may play critical roles in different subtypes of breast cancer.

We have recently demonstrated that SHP2 regulates focal adhesion kinase (FAK) in BTBC cells to promote cell polarity and migration in 2D cultures [18]. Because the SHP2 inhibition-induced loss of the mesenchymal morphology was so dramatic, we wanted to determine the effect of SHP2 silencing on cell migration for extended period of time. We have found that the SHP2 silenced cells could not completely close the monolayer wound even in 48 hours, while the control cell did it within 24 hours (Figure 2A and B). Expression of dominant-negative SHP2 also led to retardation in cell migration (Additional file 2: Figure S2A and B), confirming the importance of the SHP2 enzyme function in cell migration. Thus, inhibition of SHP2 severely retards BTBC cell migration.

In agreement with the loss of the mesenchymal morphology and migratory behavior in 2D, the SHP2silenced cells were defective in their invasive property, the hallmark of "mesenchymalness" (Figure 2C-F and Additional file 2: Figure S2C). These changes were confirmed by the reduced expression of Vimentin, FN, and Snail, the commonly used EMT markers (Figure 3A). Interestingly, proteasome inhibition restored Snail and FN expression, but not vimentin (Figure 3B). Time-course protein translation inhibition studies following Mg-132 stabilization showed that loss of SHP2 leads to rapid degradation of FN and Snail by the proteasome (Figure 3C-F). These findings suggest that SHP2 promotes FN and Snail protein stability in BTBC cells. However, our data does not show the mechanism by which SHP2 promotes the stability of FN and Snail. Future studies addressing aspect will be necessary. In case of vimentin, it seems that SHP2 promotes vimentin expression at the transcriptional level. It will also be necessary to address this point in future studies.

Ability to interact, degrade, and move through the ECM by a cancer cell is the major mechanism for the development of a malignant disease [37,38]. Matrix metalloproteinase 2 and 9 (MMP-2 and MMP-9) have been implicated as playing significant roles in enabling invasiveness and metastasis of breast cancer [31,39]. We have demonstrated that inhibition of SHP2 suppresses the expression of both proteases in BTBC cells (Figure 3G and H). In support of our findings, a previous study has also shown that SHP2 promotes the expression of MMP9 [40]. These results provide a mechanistic explanation as to how 
SHP2 promotes the invasiveness property of BTBC cells. However, our results cannot rule out the possibility of SHP2 regulating the expression and/or secretion of additional proteases to promote invasiveness. Future studies may also be needed to explore these possibilities.

For the first time, we have demonstrated that inhibition of SHP2 induces basal-to-luminal transition (BLT) in BTBC cells (Figure 4A and B). Previous studies by us and others have shown that the acini-like structures formed by isolated breast epithelial cells resemble the milk-producing structures of the normal breast. Therefore, our results are consistent with SHP2 inhibition leading to BLT. More surprising was the induction of $\mathrm{ER} \alpha$ expression (Figure 4C), which suggested that inhibition of SHP2 converts BTBC cells into hormonepositive epithelial cells.

The SHP2-inhibition induced BLT in BTBC cells was unexpected since these cells are thought to originate from the basal cells of the breast, the myoepithelial cells. One possible explanation is that the minority stem-like cell populations, also known as cancer stem cells (CSC), present in the MDA-MB-231 and the MDA-MB-468 cells [41,42], may give rise to luminal lineage cells. As in normal breast stem cells which are pluripotent and can give rise to luminal and basal lineages, the stem-like cells present in BTBC cells might have differentiated into luminal lineage upon SHP2 inhibition, leading to formation of acini-like structures in LRBM cultures. Future studies may be needed to show how inhibition of SHP2 in BTBC cells leads to differentiation of pluripotent basal cells to luminal cells.

We have demonstrated that inhibition of SHP2 converts triple-negative breast cancer cells into hormone-positive (ER $\alpha$-positive) cells that depend on estrogen signaling for growth (Figure 5A and B). We have also shown that SHP2-silenced cells are sensitive to tamoxifen treatment (Figure 5C and D). A recent study has shown that SHP2 is essential for estradiol (E2)-induced cell growth and signaling in the ER $\alpha$-positive MCF7 breast cancer cell line primarily using a small molecule-based SHP2 inhibition [43]. This report also showed cross-talk between the ER $\alpha$ and the IGF-1R-Gab2 signaling axis in which SHP2 was found to be important. It is possible that the observed inhibition of cell growth and signaling in MCF7 cells treated with the SHP2 inhibitor PHPS1 might have emanated from the loss of this cross talk. Since activation of the SHP2 enzyme function is dependent on tyrosine kinase activation and interaction of its $\mathrm{SH} 2$ domains, it is unlikely that ER $\alpha$ per se can activate SHP2 to mediate signaling. In addition, the observed differences between our findings and this report may reflect cell-context differences between the MCF7 and the SHP2-silnced BTBC cells. Nonetheless, our findings imply that SHP2 inhibition can be used to treat $\mathrm{BTBC}$ singly or in combination with the existing anti-hormone therapies.

\section{Conclusions}

In this study, we show that inhibition of SHP2 induces basal-to-luminal transition (BLT), ER $\alpha$ expression, estrogen dependency for growth, and sensitivity to anti-hormone therapy. Therefore, inhibition of SHP2 may provide a therapeutic benefit to basal-like and triple-negative breast cancer patients if specific drugs can be developed.

\section{Additional files}

\begin{abstract}
Additional file 1: Figure S1. Includes pictures of non-confluent parental, control, and SHP2 shRNA cells derived from the MDA-MB231 (A) and from the MDA-MB468 (C). Also shown in Figure S1 are pictures of confluent parental, control, and SHP2 shRNA cells derived from the MDA-MB468 cells (B). Pictures of non-confluent parental MCF-10A cells are included in all cases as a reference.

Additional file 2: Figure S2. In this supplemental data, we show that inhibition of SHP2 by dominant-negative (C459S-SHP2) expression also suppresses cell migration and matrigel invasion.
\end{abstract}

\section{Abbreviations}

BTBC: basal-like and triple-negative breast cancer; RTK: receptor tyrosine kinase; EGFR: epidermal growth factor receptor; SHP2: Src homology 2 phosphotyrosyl phosphatase; ERa: estrogen receptor alpha; the MET receptor: also known as hepatocyte growth factor receptor; EMT: epithelial-t-mesenchymal transition; MET: mesenchymal to epithelial transition; BLT: basal-to-luminal transition; LRBM: laminin-rich basement membrane.

\section{Competing interests}

The authors declare that they have no competing interests.

\section{Authors' contributions}

$\mathrm{HZ}$ carried out most of the experimental work and data organization, while YMA drafted the manuscript and prepared the final version. Both authors have read and approved the final manuscript.

\section{Acknowledgement}

This work was supported by a grant number CA124940 from the National Cancer Institute $(\mathrm{NCl})$, a component of the National Institute of Health $(\mathrm{NIH})$ to YMA. West Virginia University and $\mathrm{NIH-NCl}$ provided infrastructure and financial support, respectively. All experiments were conducted in accordance with the rules and regulations of the host institution.

Received: 5 November 2014 Accepted: 24 February 2015

Published online: 08 March 2015

\section{References}

1. Rakha EA, El-Sayed ME, Reis-Filho J, Ellis IO. Patho-biological aspects of basal-like breast cancer. Breast Cancer Res Treat. 2009;113(3):411-22.

2. Lin NU, Claus E, Sohl J, Razzak AR, Arnaout A, Winer EP. Sites of distant recurrence and clinical outcomes in patients with metastatic triple-negative breast cancer: high incidence of central nervous system metastases. Cancer. 2008;113(10):2638-45.

3. Cheang MC, Voduc D, Bajdik C, Leung S, McKinney S, Chia SK, et al. Basallike breast cancer defined by five biomarkers has superior prognostic value than triple-negative phenotype. Clin Cancer Res. 2008;14(5):1368-76.

4. Molyneux G, Geyer FC, Magnay FA, McCarthy A, Kendrick H, Natrajan R, et al. BRCA1 basal-like breast cancers originate from luminal epithelial progenitors and not from basal stem cells. Cell Stem Cell. 2010;7(3):403-17.

5. Bayraktar S, Gluck S. Molecularly targeted therapies for metastatic triplenegative breast cancer. Breast Cancer Res Treat. 2013;138(1):21-35.

6. Gastaldi S, Comoglio PM, Trusolino L. The Met oncogene and basal-like breast cancer: another culprit to watch out for? Breast Cancer Res. 2010;12(4):208

7. Nielsen TO, Hsu FD, Jensen K, Cheang M, Karaca G, Hu Z, et al. Immunohistochemical and clinical characterization of the basal-like subtype of invasive breast carcinoma. Clin Cancer Res. 2004;10(16):5367-74. 
8. Sharpe R, Pearson A, Herrera-Abreu MT, Johnson D, Mackay A, Welti JC, et al. FGFR signaling promotes the growth of triple-negative and basal-like breast cancer cell lines both in vitro and in vivo. Clin Cancer Res. 2011;17(16):5275-86.

9. Agazie YM, Hayman MJ. Molecular mechanism for a role of SHP2 in epidermal growth factor receptor signaling. Mol Cell Biol. 2003;23(21):7875-86.

10. Feng GS, Shen R, Heng HH, Tsui LC, Kazlauskas A, Pawson T. Receptorbinding, tyrosine phosphorylation and chromosome localization of the mouse SH2-containing phosphotyrosine phosphatase Syp. Oncogene. 1994;9(6):1545-50.

11. Hadari YR, Kouhara H, Lax I, Schlessinger J. Binding of Shp2 tyrosine phosphatase to FRS2 is essential for fibroblast growth factor-induced PC12 cell differentiation. Mol Cell Biol. 1998;18(7):3966-73.

12. Agazie YM, Movilla N, Ischenko I, Hayman MJ. The phosphotyrosine phosphatase SHP2 is a critical mediator of transformation induced by the oncogenic fibroblast growth factor receptor 3. Oncogene. 2003;22(44):6909-18.

13. Burks J, Agazie YM. Modulation of alpha-catenin Tyr phosphorylation by SHP2 positively effects cell transformation induced by the constitutively active FGFR3. Oncogene. 2006;25(54):7166-79.

14. Hakak Y, Hsu YS, Martin GS. Shp-2 mediates v-Src-induced morphological changes and activation of the anti-apoptotic protein kinase Akt. Oncogene. 2000;19(28):3164-71.

15. Zhou X, Agazie YM. Molecular mechanism for SHP2 in promoting HER2induced signaling and transformation. J Biol Chem. 2009;284(18):12226-34.

16. Feng GS, Hui CC, Pawson T. SH2-containing phosphotyrosine phosphatase as a target of protein-tyrosine kinases. Science. 1993;259(5101):1607-11.

17. Feng GS, Pawson T. Phosphotyrosine phosphatases with $\mathrm{SH} 2$ domains: regulators of signal transduction. Trends Genet. 1994;10(2):54-8.

18. Hartman ZR, Schaller MD, Agazie YM. The tyrosine phosphatase SHP2 regulates focal adhesion kinase to promote EGF-induced lamellipodia persistence and cell migration. Mol Cancer Res. 2013;32(35):4169-80.

19. Zhou XD, Agazie YM. Inhibition of SHP2 leads to mesenchymal to epithelial transition in breast cancer cells. Cell Death Differ. 2008;15(6):988-96.

20. Aceto N, Sausgruber N, Brinkhaus H, Gaidatzis D, Martiny-Baron G, Mazzaro $\mathrm{G}$, et al. Tyrosine phosphatase SHP2 promotes breast cancer progression and maintains tumor-initiating cells via activation of key transcription factors and a positive feedback signaling loop. Nat Med. 2012;18(4):529-37.

21. Scherr M, Chaturvedi A, Battmer K, Dallmann I, Schultheis B, Ganser A, et al. Enhanced sensitivity to inhibition of SHP2, STAT5, and Gab2 expression in chronic myeloid leukemia (CML). Blood. 2006;107(8):3279-87.

22. Premzl A, Turk V, Kos J. Intracellular proteolytic activity of cathepsin B is associated with capillary-like tube formation by endothelial cells in vitro. J Cell Biochem. 2006;97(6):1230-40.

23. Debnath J, Muthuswamy SK, Brugge JS. Morphogenesis and oncogenesis of MCF-10A mammary epithelial acini grown in three-dimensional basement membrane cultures. Methods. 2003;30(3):256-68.

24. Hollestelle A, Nagel JH, Smid M, Lam S, Elstrodt F, Wasielewski M, et al. Distinct gene mutation profiles among luminal-type and basal-type breast cancer cell lines. Breast Cancer Res Treat. 2010;121(1):53-64.

25. Neve RM, Chin K, Fridlyand J, Yeh J, Baehner FL, Fevr T, et al. A collection of breast cancer cell lines for the study of functionally distinct cancer subtypes. Cancer Cell. 2006;10(6):515-27.

26. O'Connor PM, Jackman J, Bae I, Myers TG, Fan S, Mutoh M, et al. Characterization of the p53 tumor suppressor pathway in cell lines of the National Cancer Institute anticancer drug screen and correlations with the growth-inhibitory potency of 123 anticancer agents. Cancer Res. 1997;57(19):4285-300.

27. Filmus J, Pollak MN, Cailleau R, Buick RN. MDA-468, a human breast cancer cell line with a high number of epidermal growth factor (EGF) receptors, has an amplified EGF receptor gene and is growth inhibited by EGF. Biochem Biophys Res Commun. 1985;128(2):898-905.

28. Zhou X, Coad J, Ducatman B, Agazie YM. SHP2 is up-regulated in breast cancer cells and in infiltrating ductal carcinoma of the breast, implying its involvement in breast oncogenesis. Histopathology. 2008;53(4):389-402.

29. Chavez KJ, Garimella SV, Lipkowitz S. Triple negative breast cancer cell lines: one tool in the search for better treatment of triple negative breast cancer. Breast Dis. 2010;32(1-2):35-48.

30. Jinga DC, Blidaru A, Condrea I, Ardeleanu C, Dragomir C, Szegli G, et al. MMP-9 and MMP-2 gelatinases and TIMP-1 and TIMP-2 inhibitors in breast cancer: correlations with prognostic factors. J Cell Mol Med. 2006;10(2):499-510.

31. Kousidou OC, Roussidis AE, Theocharis AD, Karamanos NK. Expression of MMPs and TIMPs genes in human breast cancer epithelial cells depends on cell culture conditions and is associated with their invasive potential Anticancer Res. 2004:24(6):4025-30.

32. Hartman Z, Zhao H, Agazie YM. HER2 stabilizes EGFR and itself by altering autophosphorylation patterns in a manner that overcomes regulatory mechanisms and promotes proliferative and transformation signaling. Oncogene. 2013:32(35):4169-80.

33. Rakha EA, Ellis IO. Triple-negative/basal-like breast cancer: review. Pathology. 2009:41(1):40-7.

34. Rakha EA, El-Sayed ME, Green AR, Lee AH, Robertson JF, Ellis IO. Prognostic markers in triple-negative breast cancer. Cancer. 2007;109(1):25-32.

35. Ahmed Z, Lin CC, Suen KM, Melo FA, Levitt JA, Suhling K, et al. Grb2 controls phosphorylation of FGFR2 by inhibiting receptor kinase and Shp2 phosphatase activity. J Cell Biol. 2013;200(4):493-504.

36. Li J, Reed SA, Johnson SE. Hepatocyte growth factor (HGF) signals through SHP2 to regulate primary mouse myoblast proliferation. Exp Cell Res. 2009;315(13):2284-92.

37. Steeg PS. Tumor metastasis: mechanistic insights and clinical challenges. Nat Med. 2006;12(8):895-904.

38. Weigelt $B$, Peterse $J L$, van 't Veer $L$. Breast cancer metastasis: markers and models. Nat Rev Cancer. 2005;5(8):591-602.

39. Kohrmann A, Kammerer U, Kapp M, Dietl J, Anacker J. Expression of matrix metalloproteinases (MMPs) in primary human breast cancer and breast cancer cell lines: New findings and review of the literature. BMC Cancer. 2009;9:188.

40. Wang FM, Liu HQ, Liu SR, Tang SP, Yang L, Feng GS. SHP-2 promoting migration and metastasis of MCF-7 with loss of E-cadherin, dephosphorylation of FAK and secretion of MMP-9 induced by IL-1 beta in vivo and in vitro. Breast Cancer Res Treat. 2005;89(1):5-14.

41. Engelmann $\mathrm{K}$, Shen $\mathrm{H}$, Finn OJ. MCF7 side population cells with characteristics of cancer stem/progenitor cells express the tumor antigen MUC1. Cancer Res. 2008;68(7):2419-26.

42. Fillmore CM, Kuperwasser C. Human breast cancer cell lines contain stemlike cells that self-renew, give rise to phenotypically diverse progeny and survive chemotherapy. Breast Cancer Res. 2008;10(2):R25.

43. Li J, Kang Y, Wei L, Liu W, Tian Y, Chen B, et al. Tyrosine phosphatase Shp2 mediates the estrogen biological action in breast cancer via interaction with the estrogen extranuclear receptor. PLoS One. 2014;9(7):e102847.

\section{Submit your next manuscript to BioMed Central and take full advantage of:}

- Convenient online submission

- Thorough peer review

- No space constraints or color figure charges

- Immediate publication on acceptance

- Inclusion in PubMed, CAS, Scopus and Google Scholar

- Research which is freely available for redistribution 